\title{
Koala retrovirus epidemiology, transmission mode, pathogenesis, and host immune response in koalas (Phascolarctos cinereus): a review
}

\author{
Mohammad Enamul Hoque Kayesh ${ }^{1,2} \cdot$ Md Abul Hashem $^{1,3} \cdot$ Kyoko Tsukiyama-Kohara $^{1}$ (i)
}

Received: 2 June 2020 / Accepted: 7 July 2020 / Published online: 8 August 2020

(c) Springer-Verlag GmbH Austria, part of Springer Nature 2020

\begin{abstract}
Koala retrovirus (KoRV) is a major threat to koala health and conservation. It also represents a series of challenges across the fields of virology, immunology, and epidemiology that are of great potential interest to any researcher in the field of retroviral diseases. KoRV is a gammaretrovirus that is present in both endogenous and exogenous forms in koala populations, with a still-active endogenization process. KoRV may induce immunosuppression and neoplastic conditions such as lymphoma and leukemia and play a role in chlamydiosis and other diseases in koalas. KoRV transmission modes, pathogenesis, and host immune response still remain unclear, and a clear understanding of these areas is critical for devising effective preventative and therapeutic strategies. Research on KoRV is clearly critical for koala conservation. In this review, we provide an overview of the current understanding and future challenges related to KoRV epidemiology, transmission mode, pathogenesis, and host immune response and discuss prospects for therapeutic and preventive vaccines.
\end{abstract}

\section{Introduction}

Koala retrovirus (KoRV) is a source of great concern to conservationists, as well as a source of great interest to virologists, immunologists, and epidemiologists. KoRV threatens koala (Phascolarctos cinereus) populations, both in the wild in Australia and in zoos around the world. Koala conservation has become an even more pressing issue of late as populations of Australia's iconic marsupial species have been devastated in recent bush fires in that country, heightening the need to prioritize efforts against KoRV. However, lessons learned in the fight against KoRV will be applicable far beyond the field of koala conservation.

Handling Editor: Zhongjie Shi.

Kyoko Tsukiyama-Kohara

kkohara@vet.kagoshima-u.ac.jp

1 Transboundary Animal Diseases Centre, Joint Faculty of Veterinary Medicine, Kagoshima University, 1-21-24 Korimoto, Kagoshima 890-0065, Japan

2 Department of Microbiology and Public Health, Faculty of Animal Science and Veterinary Medicine, Patuakhali Science and Technology University, Barishal 8210, Bangladesh

3 Department of Health, Chattogram City Corporation, Chattogram 4000, Bangladesh
$\mathrm{KoRV}$ is a gammaretrovirus belonging to the family Retroviridae and was first identified in 1988 in a leukemic koala [1]. Subsequently, the KoRV genome was fully sequenced for the first time in 2000 [2]. KoRV has the typical morphology of a gammaretrovirus, with spherically shaped virions ranging from $80-100 \mathrm{~nm}$ in diameter [3]. KoRV has a positive-sense, single-stranded RNA genome approximately $8.4 \mathrm{~kb}$ in size, containing gag, pol, and env genes with long terminal repeat (LTR) regions at the $5^{\prime}$ and $3^{\prime}$ ends [2, 4, 5]. The KoRV genome encodes a reverse transcriptase and structural proteins such as the main core protein $\mathrm{p} 27 \mathrm{Gag}$ and the envelope proteins gp70 and p15E [6].

KoRV is closely related genetically to gibbon-ape leukemia virus (GaLV) [2, 4], feline leukemia virus (FeLV), and porcine endogenous retrovirus (PERV) [3]. KoRV has been linked to immunosuppression, chlamydiosis, lymphoma, and leukemia and leads to poor outcomes for infected individuals [4, 7-10]. Progress in KoRV-related research may therefore shed light on issues that need to be tackled for any retrovirus.

A further interesting feature of KoRV is its existence in both exogenous and endogenous forms, in contrast to human endogenous retroviruses (HERVs), which exist entirely in the human genome but show few associations with pathologies. The endogenization process for KoRV started relatively recently (approximately 22,000 to 49,000 years ago) [11] and appears to be ongoing; KoRV thus presents a great opportunity to study the virus endogenization process in real 
time. By contrast, other mammalian retrovirus infections date back over millions of years of evolutionary history, during which initially exogenous retroviruses were integrated into the host germ line and became endogenous viruses that were solely transmitted vertically from parents to offspring [12]. KoRVs, on the other hand, can be transmitted both vertically and horizontally [5, 7, 13-16].

So far, nine subtypes of KoRV have been isolated (KoRVA to KoRV-I; Table 1) [17]. This classification is based on sequence differences in the receptor-binding domain of the envelope gene [18, 19]. Of the nine subtypes, KoRV-A and KoRV-B are currently the most extensively characterized [7, 9]. KoRV-A is transmitted vertically, whereas KoRV-B is transmitted both vertically and horizontally [4, 9, 17]. KoRV-A and KoRV-B utilize different entry receptors: phosphate transporter (PiT1) in the case of KoRV-A and thiamine transporter 1 (ThTR1) in the case of KoRV-B [7, 20]. Also, many recombinant KoRV (recKoRV) variants have been recognized in koalas [15, 21, 22].

The prevalence of KoRV varies depending on the geographical location of a particular population [13]. Variations in KoRV proviral loads in koala genomes have been reported by us and others [5, 13, 16, 23-25]. These reports are indicative of the ongoing endogenization process that KoRV is undergoing.

All vertebrates tested to date harbor endogenous retroviruses [26], which are genetic traces of past infections in which the viral genome was endogenized and then rendered inactive due to substitution mutations and deletions [27, 28]. In contrast, genetic analysis has revealed that endogenous KoRV contains a full-length replication-competent genome showing an infection pattern similar to that of exogenous retroviruses [29].

Although the pathogenesis of KoRV infections in koalas is poorly understood, KoRV-associated pathologies in koalas are considered a major threat to koala health and conservation. KoRV has been linked to neoplastic diseases, including lymphoma and leukemia in wild and captive koalas, which are associated with high mortality $[4,7,30]$.

As the information summarized above makes clear, much research has been undertaken on KoRV from a variety of perspectives (ranging across wildlife conservation, epidemiology, virology, microbiology, and immunology); however, our knowledge in all of these spheres is incomplete. An overview of what is known-and what is not knownabout KoRV is particularly timely and will be of wide interest across the scientific community.

In this review, we outline the state of existing knowledge on KoRV and outline the challenges that remain. A proper understanding of the epidemiology and pathogenesis of KoRV infection and host immune responses against KoRV infection are critical for the development of effective preventative and therapeutic strategies for this virus. We focus on the epidemiology, transmission routes, pathogenesis, host immune response, and the issue of vaccine development in our survey of this interesting and challenging viral infection of koalas.

\section{Epidemiology of KoRV}

KoRV is now endemic in both wild and captive koalas and represents a major threat to koala health [7, 9, 23, 31-33]. KoRV shows variations in its prevalence depending on the geographical location of the population and the virus subtype [13, 25, 33-35] (Fig. 1).

Epidemiolocal research on KoRV in the wild has tended to divide koalas broadly into two populations on the island continent of Australia: a northern population with habitats in Queensland and northern New South Wales and a southern population with habitats in Victoria and South Australia. Within the northern population, the prevalence of KoRV provirus is regarded as $100 \%$, with every reported case of testing yielding positive evidence of the presence of a provirus $[4,9,13,33]$ (Table 2$)$. The southern population

Table 1 A summary of KoRV transmission modes

\begin{tabular}{llll}
\hline Subtype & Endogenous or exogenous & Transmission mode & Study area \\
\hline KoRV-A & Endogenous & Vertical & Wild and captive Australian koala populations [4, 9, \\
KoRV-B & $\begin{array}{c}\text { Exogenous, highly prevalent and associated } \\
\text { with diseases }\end{array}$ & Both horizontal and vertical & $\begin{array}{c}\text { Wild koala populations in Australia [9, 17], koalas } \\
\text { in a US zoo [7], koalas in a Japanese zoo [14, 24] }\end{array}$ \\
KoRV-C & Exogenous with low prevalence & Horizontal and vertical & US zoo [57], Japanese zoo [16] \\
KoRV-D & Exogenous & Horizontal & Wild koala populations in Australia [15, 17] \\
KoRV-E & Exogenous with low prevalence & Horizontal & Wild koala in Australia [15], koalas in a US zoo [73] \\
KoRV-F & Exogenous & Horizontal & Wild koala populations in Australia [17, 18] \\
KoRV-G & Ongoing exogenous with low prevalence & Horizontal & Wild koala populations in Australia [18] \\
KoRV-H & Ongoing exogenous with low prevalence & Horizontal & Wild koala populations in Australia [18] \\
KoRV-I & Ongoing exogenous and low prevalent & Horizontal & Wild koala populations in Australia [18] \\
\hline
\end{tabular}




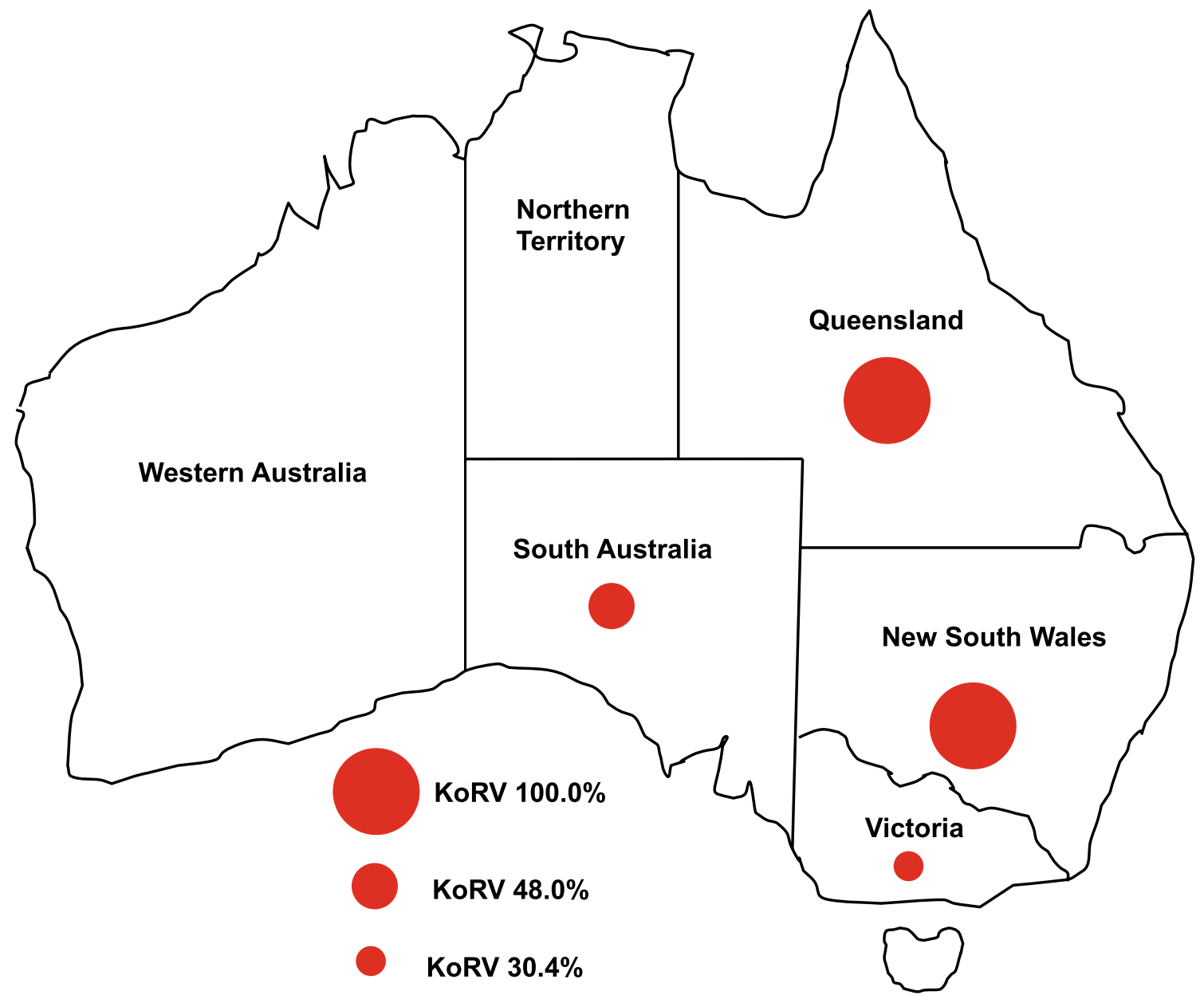

Fig. 1 The geographical distribution of KoRV provirus in free-ranging koala populations in Australia. The KoRV provirus prevalence data shown on the map have been summarized from Table 2. The prevalence of KoRV provirus was shown to be $100 \%$ in koala popula-

previously showed a much lower prevalence, reportedly ranging from 14.8 to $25 \%$ [8, 13, 34]; however, the three most recent studies have shown markedly higher figures, ranging from 42.4 to $99.0 \%$ [25, 33, 35] (Table 2).

Genetic diversity has also been addressed in other recent studies. The southern population appears to show lower absolute proviral copy numbers than the northern population [33]. A possible explanation advanced for this phenomenon concerns the relatively high replication efficiency of subtypes other than KoRV-A in the southern population [35]. Most of the data obtained so far concern KoRV subtypes $\mathrm{A}$ and $\mathrm{B}$, and the data are far from complete for subtypes $\mathrm{C}$ to I. However, subtype expression has been addressed in one recent study, in which KoRV-A, -B, -D and -F were found to be the major subtypes present in wild populations and KoRV-D was found to be the significantly most prevalent subtype, with 10- and 100-fold greater expression than KoRV-A and KoRV-F, respectively [17]. tions of Queensland (805/805) and New South Wales (100/100), vs. $48.0 \%$ (268/558) and 30.4\% (255/839) in koala populations of South Australia and Victoria, respectively. This map was generated from maps of Australia that are available through the internet

The endogenous subtype, KoRV-A, has a prevalence rate of $100 \%$ in the northern population $[4,9,13]$. However, KoRV-A is also reportedly spreading within the southern population [13], with prevalence reaching $42.4 \%$ and $65.3 \%$ at the respective sampling points in the states of Victoria and South Australia, but these findings were not accompanied by the detection of KoRV-B [33] (Table 3). However, the prevalence of KoRV-B may be increasing in the northern population; a previous figure was given as $24 \%$ [9], with $48.5 \%$ as the most recently reported prevalence of KoRV-B in the northern population [35] (Table 3).

The data reviewed above were obtained exclusively from wild populations, but some data are also available for zoo populations. We have previously reported a KoRV-A prevalence of $100 \%$ and a KoRV-B prevalence ranging from 50 to $63.6 \%$ in Japanese zoo populations (at Kobe Oji Zoo, Saitama Children's Zoo, and Hirakawa Zoological Park) [24]. The origins of these populations were not entirely 
Table 2 Variation of KoRV prevalence in northern (Queensland and New South Wales) and southern (Victoria and South Australia) koala populations in Australia

\begin{tabular}{|c|c|c|c|c|}
\hline \multirow[t]{2}{*}{ Reference } & \multicolumn{4}{|c|}{ KoRV prevalence $(\%)$} \\
\hline & Queensland & New South Wales & Victoria & South Australia \\
\hline [5] & $\begin{array}{l}\mathrm{n}=98 \\
98 / 98(100.0)\end{array}$ & - & $\begin{array}{l}\text { Ballart }(\mathrm{n}=5) \\
3 / 5(60.0) \\
\text { Raymond Island }(\mathrm{n}=17) \\
5 / 17(29.4)\end{array}$ & $\begin{array}{l}\text { Kangaroo Island }(\mathrm{n}=26 \\
0 / 26(0.0)\end{array}$ \\
\hline [13] & $\begin{array}{l}n=277 \\
277 / 277(100.0)\end{array}$ & $\begin{array}{l}\mathrm{n}=100 \\
100 / 100(100.0)\end{array}$ & $\begin{array}{l}\text { Mainland Victoria }(\mathrm{n}=89) \\
65 / 89(73.0) \text {; } \\
\text { Four Victoria Islands }(\mathrm{n}=80) \\
22 / 80(26.6)\end{array}$ & $\begin{array}{l}\text { Kangaroo Island }(n=162) \\
24 / 162(14.8) \\
-\end{array}$ \\
\hline [34] & - & - & $\begin{array}{l}\mathrm{n}=648 \\
160 / 648(24.7)\end{array}$ & - \\
\hline [8] & $\begin{array}{l}\mathrm{n}=36 \\
36 / 36(100.0)\end{array}$ & - & - & - \\
\hline [9] & $\begin{array}{l}\mathrm{n}=290 \\
290 / 290(100.0)\end{array}$ & - & - & - \\
\hline [33] & - & - & - & $\begin{array}{l}\text { Kangaroo Island } \\
(\mathrm{n}=170) \\
72 / 170(42.4) \\
\text { Mount Lofty Ranges }(\mathrm{n}=75) \\
49 / 75(65.3)\end{array}$ \\
\hline [35] & $\begin{array}{l}\mathrm{n}=33 \\
33 / 33(100.0)\end{array}$ & - & - & $\begin{array}{l}n=28 \\
27 / 28(96.4)\end{array}$ \\
\hline [25] & $\begin{array}{l}\mathrm{n}=71 \\
71 / 71(100.0)\end{array}$ & - & - & $\begin{array}{l}\mathrm{n}=97 \\
96 / 97(99.0)\end{array}$ \\
\hline
\end{tabular}

clear. However, the three koalas targeted at one of these zoos (Hirakawa Zoological Park) had been born at a theme park in Queensland; hence, the findings that all three animals were KoRV-A positive while only two of three animals were KoRV-B positive are unsurprising [24]. In a recent study on coinfection in Japanese zoo-dwelling koalas, we found two animals to be positive for KoRV-A, KoRV-B, and KoRV-C [16]. Furthermore, in matching findings reported by us and other researchers, infectious virions have been produced from peripheral blood mononuclear cells (PBMCs) isolated from KoRV-positive koalas when co-cultured with HEK293T cells [23, 31]. In addition to human cells, cells from rats, cats, and mink can reportedly be infected with KoRV in vitro, although mouse cells cannot [6, 14, 20, 29]. However, the data from in vivo studies are limited; live rats reportedly can be infected with KoRV, but the pathogenicity remains unclear [6]. The data for zoo populations still falls short of the data available for wild populations, and the prevalence of KoRV subtypes C-I in koalas in Japanese zoo populations is still unclear.

\section{Transmission mode of KoRV}

KoRV shows some resemblance to other retroviruses in terms of transmission. For example, HIV types 1 and 2 are known to jump species and infect new hosts [36, 37].
Similarly, KoRV is a potentially fatal gammaretrovirus that crossed the species barrier into koalas. The species from which the virus jumped into koalas is unknown. One candidate is a native Australian rodent, the grassland melomys (Melomys burtoni) [38, 39], and bats have also been suggested [40]. The argument for bats has been strengthened by the recently reported presence of KoRV-related gammaretroviruses in Australian and Asian bats, which suggests that bats may be an important reservoir of exogenous KoRVrelated gammaretroviruses [40].

The endogenous form (KoRV-A) has infected germ cells and become a permanent resident of the koala genome. KoRV-A sequences are usually highly conserved among various isolates and lack the genetic diversity of an exogenous retrovirus [41]. However, researchers have observed that KoRV integrates into the koala genome at many locations, mostly unique to each individual koala, which suggests that integration is still recent and not uniform and fixed in the koala population [11, 21, 42, 43]. Transmission is understood to be vertical (dam-to-offspring) for the endogenous form [5, 9], but the mechanisms of horizontal transmission are not fully clear. Entry receptors have been reported for KoRV-A and KoRV-B [7, 20], but not for the other subtypes (KoRV-C to KoRV-I).

Different transmission rates for KoRV-B — an exogenous subtype-have been reported. An annual transmission rate 
Table 3 Variation of KoRV subtype prevalence in northern and southern koala populations in Australia

\begin{tabular}{|c|c|c|c|}
\hline \multirow[t]{2}{*}{ Reference } & \multicolumn{3}{|c|}{ KoRV subtype prevalence (\%) } \\
\hline & Queensland & Victoria & South Australia \\
\hline [34] & - & $\begin{array}{l}\mathrm{n}=166 \\
\text { KoRV-A } \\
141 / 166(88.8)\end{array}$ & - \\
\hline [8] & $\begin{array}{l}\mathrm{n}=36 \\
\text { KoRV-A } \\
36 / 36(100.0) \\
\text { KoRV-B } \\
\text { 9/36 (25.0) }\end{array}$ & - & - \\
\hline [9] & $\begin{array}{l}\mathrm{n}=290 \\
\text { KoRV-A } \\
290 / 290(100.0) \\
\text { KoRV-B } \\
70 / 290(24.1)\end{array}$ & - & - \\
\hline [33] & - & - & $\begin{array}{l}\text { Kangaroo Island } \\
\mathrm{n}=170 \\
\text { KoRV-A } \\
72 / 170(42.4) \\
\text { KoRV-B } \\
\text { 0/170 (0.0) } \\
\text { Mount Lofty Ranges } \\
\mathrm{n}=75 \\
\text { KoRV-A } \\
49 / 75(65.3) \\
\text { KoRV-B } \\
\text { 0/75 (0.0) }\end{array}$ \\
\hline [35] & $\begin{array}{l}\mathrm{n}=33 \\
\text { KoRV-A } \\
33 / 33(100.0) \\
\text { KoRV-B } \\
16 / 33(48.5)\end{array}$ & - & $\begin{array}{l}\mathrm{n}=28 \\
\text { KoRV-A } \\
27 / 28(96.4) \\
\text { KoRV-B } \\
0 / 28(0.0)\end{array}$ \\
\hline
\end{tabular}

of 3\% has been reported for adult-to-adult koala contact [9]. We previously reported on a case of sire-to-offspring transmission, differing from other reports where the transmission was from dam to offspring [7, 9]. Interestingly, we recently found a KoRV-B-negative offspring born of KoRV-B-positive parents [16]. Taken together, these results support the existence of a horizontal transmission mode for KoRV-B; however, little is known about the transmission modes for other subtypes, which require further investigation in order to achieve a complete understanding of how KoRV is transmitted. The current state of knowledge on KoRV transmission modes for different subtypes is summarized in Table 1.

\section{Pathogenesis of KoRV}

Retroviruses are known to cause a range of diseases in their hosts, including neoplasia and central nervous system and immunosuppressive disorders [44-46]. Such causative associations have been postulated for KoRV, but the exact causal relationships between KoRV and a range of clinical diseases have yet to be established. Although direct associations of KoRV and immunosuppression and neoplasia are not well documented, it has been shown that the KoRV viral load positively correlates with leukemia, lymphoma, and chlamydial disease in koalas [4, 47, 48], and the association with chlamydial disease in particular is regarded as a significant long-term threat to the survival of the species [4, 7-9].

KoRV-B was recently shown to be more pathogenic than KoRV-A and to have a significant association with chlamydial infection, although the exact molecular mechanism is not clear [8]. KoRV-B is also associated with neoplasia in koalas [7]. KoRV-B is an exogenous form of the virus, and exogenous KoRV infection has attracted recent attention for its role in periodontal disease, with speculation that exogenous KoRV may modulate the koala's immune response when they contract bacterial infections within the oral cavity [49].

KoRV-associated neoplasia may cause up to $60 \%$ of koala mortalities in captivity and at least $5 \%$ in the wild [2]. A high level of viremia is correlated with an increased risk of neoplasia and immunosuppression in koalas [47]. Lymphoma was recently reported for the first time in a South Australian KoRV-A-positive female koala, which was also affected by severe reproductive chlamydiosis [50].

As outlined above, koala populations in northern and southern Australia show differences in KoRV subtype prevalences and KoRV viral loads. This presents an interesting opportunity to investigate geographical variations in pathogenesis according to the characterization of viral disease in different populations; however, research on the full implications for lymphoma and chlamydial disease are still ongoing. Viral load was found to correlate positively with chlamydial disease severity in a southern population in a recent investigation looking for such northern-versus-southern patterns in viral load and disease pathology within Australia [48]. The same study also revealed that proviral and viral loads were positively correlated with lymphocyte and metarubricyte counts, and that viral load was positively correlated with splenic lymphoid area. These findings are suggestive of KoRV-induced oncogenesis [48].

Recent findings have enhanced our understanding of KoRV-induced pathogenesis in koalas, but much remains to be elucidated on the role of KoRV in neoplasms such as lymphoma and chlamydiosis. Extensive further investigations are required.

\section{Immune response against KoRV infection in koalas}

Without a full understanding of how a host responds to a particular pathogen, prophylactic and therapeutic interventions cannot be developed for any disease, and immunological studies are essential to achieve such an understanding $[51,52]$. The current situation with KoRV provides a good illustration of this point. Much remains unknown about how 
koalas respond to KoRV, and studies of the immunomodulatory effects of KoRV in koalas are limited in number. This presents a challenge for those seeking to conserve koala populations. However, there have been promising developments in this field of research, as outlined below.

Whole-genome sequencing has recently been achieved for koalas, and this is expected to greatly facilitate studies of immune responses in this species [22]. Fiebig et al. [6] observed increases in expression of interleukin-6 (IL-6) and IL-10 in human PBMCs incubated with KoRV, and these increases are suggestive of inflammation and immunosuppression, respectively, as shown in Fig. 2. The KoRV transmembrane envelope protein $\mathrm{p} 15 \mathrm{E}$ has been found to contain an immunosuppressive domain that is highly conserved in different retroviruses [53]. The sequence of the KoRV p15E protein is highly conserved among different KoRV subtype proviruses, including those reported to be exogenous [54].

In a study with 16 koalas, Fiebig et al. observed that koalas did not produce antibodies against KoRV-A [55], but in a recent study using 235 koalas, Quigley et al. analyzed the antibody response and found that most koalas produced antiKoRV antibodies [56]. This finding is very important for developing a therapeutic KoRV vaccine. In a previous study, we detected a high copy number of KoRV RNA $(102,473$ copies $/ \mathrm{mL}$ ) in the plasma of one koala, which then became negative when tested again several months later [23]. This might have been due to the induction of immune response [56].

Full-length sequences have been reported for important immune response genes in koalas, and diverse major histocompatibility complex (MHC) genes with 34 SNPs have been identified in a study using transcriptome analysis [57]. CD4 and CD8 $\beta$ expression were found to be downregulated when expression profiles of several immune genes were investigated in mitogen-stimulated lymphocytes isolated from KoRV-A-positive koalas (Fig. 2) [58]. The $\mathrm{CD} 4: \mathrm{CD} 8$ gene expression ratio is a potential biomarker for immunological evaluation [59, 60]. In a recent study, Maher et al. characterized the immune response to KoRV infection and found immunophenotypic changes in free-ranging Victorian koalas with significantly lower levels of IL17A and IFN- $\gamma$ gene expression accompanying a significantly decreased ratio of CD4:CD8 gene expression relative to that seen in KoRV-negative koalas (Fig. 2) [10]. Decreased levels of IL17A and IFN- $\gamma$ may render koalas more susceptible to bacterial and chlamydial infections, respectively [10]. Epitopes on the KoRV envelope protein that can be recognized by antibodies have been identified recently, showing that anti-KoRV IgG can be induced and decrease the viral load of KoRV [61].

Thus, recent advances have been made in our understanding of the immune response to KoRV infection in koalas. These advances will play a crucial role on the road to developing an effective vaccine that can protect koalas.

\section{Vaccines}

Currently, no licensed vaccine or effective treatment is available for KoRV or its associated neoplastic diseases. The majority of koalas are infected with KoRV, and therapeutic vaccines urgently need to be developed as we seek to curb the fatalities in koalas. A range of studies have demonstrated that therapeutic vaccines can enhance immunogenicity, reducing the viral or bacterial load in both human and animal infections, and that they can reduce pathogen-associated morbidity and mortality [62-66].

Effective prophylactic vaccines against the exogenous subtypes are also urgently needed. Virus-neutralizing antibody responses and $\mathrm{T}$ cell responses are both important for vaccines that can effectively protect against retroviral infection [67]. The recent failure of an experimental AIDS vaccine in a phase IIb study highlights the need for a balanced immune response consisting of not only cellular immunity but also a broad and potent antibody
Fig. 2 A schematic representation of the immune response to KoRV infection. Entry of KoRV into PBMCs induces the upregulation of IL-10 and IL-6, which may induce immunosuppression and inflammation. KoRV infection can also decrease IL-17A, IFN- $\gamma$ and the CD4:CD8 ratio, which can also induce immunosuppression

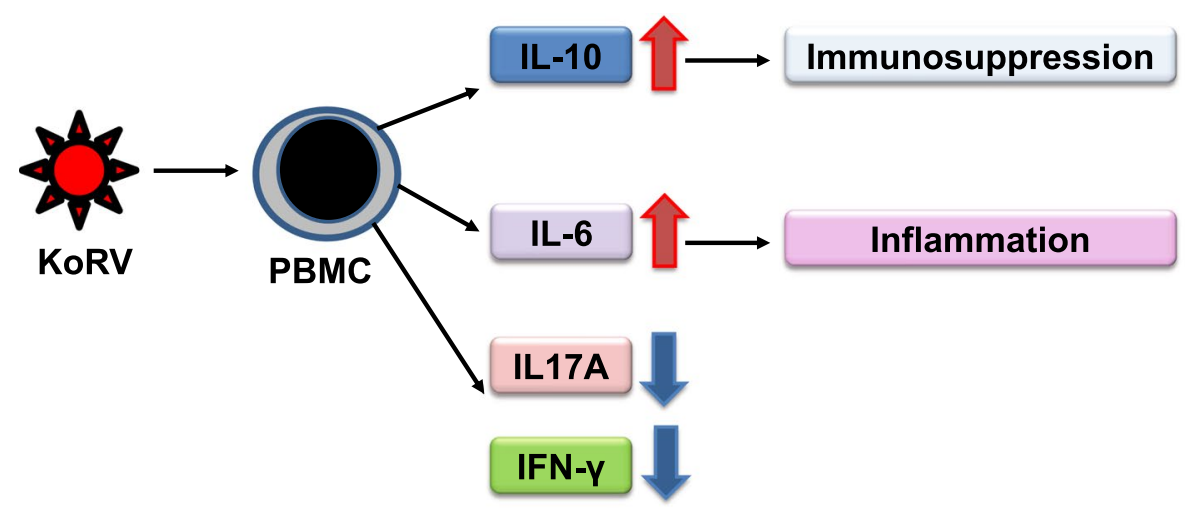

CD4:CD8 ratio
Immunosuppression 
response, which is essential for preventing retrovirus infection [68]. Inducible neutralizing antibodies show great promise for preventing retrovirus infections, and these antibodies have envelope proteins as their main targets [69]. Notably, an effective vaccine against FeLV infection is available, and this vaccine contains an envelope protein as an immunogen [70]. Neutralizing antibodies against KoRV were reportedly raised after immunization with a recombinant transmembrane envelope protein rp15E [6]. Specifically, in that study, neutralizing antibodies were raised in significantly higher numbers after immunization with KoRV gp70 than rp15E. Furthermore, PERV was neutralized with antisera against $\mathrm{p} 15 \mathrm{E}$ and gp70 of KoRV and PERV [6]. Therefore, it seems likely that KoRV envelope proteins can be utilized for the development of effective preventive or therapeutic vaccines for protecting koalas from infection. Currently, studies associated with the development of vaccines against KoRV are underway $[30,61,71]$. In one of these studies, a recombinant KoRV envelope protein vaccine candidate induced a significant humoral immune response and production of neutralizing antibodies in both KoRV-infected and KoRVfree koalas [61]. However, the abundant genetic diversity of KoRV encompasses nine KoRV subtypes [18], and this is a potentially critical point to consider for researchers seeking to prevent and control KoRV through vaccination. A number of recent studies have thrown further light on KoRV subtypes and have implications for research on KoRV vaccines. Hobbs et al. found KoRV-D and KoRV-E subtypes to be defective viruses, with significant deletions in the gag and pol genes [15]. Notably, recKoRV variants have been widely reported [15, 21, 22], and these may have been generated by recombination events due to retrotransposition or exogenous infection with KoRV [15]. Löber et al. demonstrated that recombination with an ancient koala retroelement disables KoRV, and this occurs frequently at an early point in the invasion process [21]. However, events involving recombination between endogenous KoRV and exogenous variants may occur, and researchers cannot rule out the possibility of a new variant with greater virulence emerging as the result of such recombination events.

Vaccine development necessitates the establishment of animal models of infection, but none have been established so far. To solve this problem, we are investigating tupaias (Tupaia belangeri) as a model of KoRV. We have successfully infected tupaia cells with KoRV [24]. This research provides evidence that tupaias are a potential new animal model for several viral infections [72]. Although more studies are required, the initial vaccineinduced specific immune responses appear promising and offer hope that koalas can be protected from KoRV infection.

\section{Conclusions}

The current knowledge about KoRV is not complete, and progress has been hampered by, for example, an insufficient understanding of the immune response in koalas due to a lack of analytical tools. However, the recent publication of the whole koala genome sequence may lead to a range of new analytical tools such as antibodies and PCR assays, and the prospects of achieving a better understanding of KoRV pathogenesis and the immune response in koalas are good. Such understanding will be crucial for the development of more-effective therapeutic and prophylactic strategies. Additional research is needed to throw light on areas such as KoRV genetic diversity, the evolution of endogenous and exogenous subtypes, and the associations of subtypes with diseases that threaten long-term koala survival. As such research progresses on a range of fronts, we can anticipate remedying the current situation where no treatment or vaccine is available for KoRV infection. The development of urgently needed prophylactic and therapeutic strategies against KoRV will clearly benefit koala conservation, but the knowledge gained could be applicable for scientists seeking to combat similar retroviral disease across a wider range of species.

Acknowledgements We would like to thank Mr. Henry Smith of the Joint Faculty of Veterinary Medicine, Kagoshima University, Japan, for his help in English editing of this manuscript. This work was supported by grants from the Ministry of Education, Science, and Culture, Japan.

\section{Compliance with ethical standards}

Conflict of interest The authors declare that they have no competing financial interests.

Ethical approval This study was done in accordance with institutional committee protocols.

\section{References}

1. Canfield PJ, Sabine JM, Love DN (1988) Virus particles associated with leukaemia in a koala. Aust Vet J 65:327-328

2. Hanger JJ, Bromham LD, McKee JJ, O'Brien TM, Robinson WF (2000) The nucleotide sequence of Koala (Phascolarctos cinereus) retrovirus: a novel type $\mathrm{C}$ endogenous virus related to Gibbon ape leukemia virus. J Virol 74:4264-4272

3. Denner J, Young PR (2013) Koala retroviruses: characterization and impact on the life of koalas. Retrovirology 10:108

4. Tarlinton R, Meers J, Hanger J, Young P (2005) Real-time reverse transcriptase PCR for the endogenous koala retrovirus reveals an association between plasma viral load and neoplastic disease in koalas. J Gen Virol 86:783-787

5. Tarlinton RE, Meers J, Young PR (2006) Retroviral invasion of the koala genome. Nature 442:79-81 
6. Fiebig U, Hartmann MG, Bannert N, Kurth R, Denner J (2006) Transspecies transmission of the endogenous koala retrovirus. J Virol 80:5651-5654

7. Xu W, Stadler CK, Gorman K et al (2013) An exogenous retrovirus isolated from koalas with malignant neoplasias in a US zoo. Proc Natl Acad Sci USA 110:11547-11552

8. Waugh CA, Hanger J, Loader J, King A, Hobbs M, Johnson R, Timms P (2017) Infection with koala retrovirus subgroup B (KoRV-B), but not KoRV-A, is associated with chlamydial disease in free-ranging koalas (Phascolarctos cinereus). Sci Rep 7:134

9. Quigley BL, Ong VA, Hanger J, Timms P (2018) Molecular dynamics and mode of transmission of koala retrovirus as it invades and spreads through a wild Queensland koala population. J Virol 92:e01871-e1917

10. Maher IE, Patterson J, Curnick M, Devlin J, Higgins DP (2019) Altered immune parameters associated with koala retrovirus (KoRV) and chlamydial infection in free ranging Victorian koalas (Phascolarctos cinereus). Sci Rep 9:11170

11. Ishida Y, Zhao K, Greenwood AD, Roca AL (2015) Proliferation of endogenous retroviruses in the early stages of a host germ line invasion. Mol Biol Evol 32:109-120

12. Gifford R, Tristem M (2003) The evolution, distribution and diversity of endogenous retroviruses. Virus Genes 26:291-315

13. Simmons GS, Young PR, Hanger JJ, Jones K, Clarke D, McKee JJ, Meers J (2012) Prevalence of koala retrovirus in geographically diverse populations in Australia. Aust Vet J 90:404-409

14. Shojima T, Yoshikawa R, Hoshino S et al (2013) Identification of a novel subgroup of koala retrovirus from koalas in Japanese Zoos. J Virol 87:9943-9946

15. Hobbs M, King A, Salinas R et al (2017) Long-read genome sequence assembly provides insight into ongoing retroviral invasion of the koala germline. Sci Rep 7:15838

16. Hashem MA, Maetani F, Kayesh MEH et al (2020) Transmission of koala retrovirus from parent koalas to a joey in a Japanese zoo. J Virol 94:e00019-20

17. Quigley BL, Phillips S, Olagoke O, Robbins A, Hanger J, Timms $P(2019)$ Changes in endogenous and exogenous koala retrovirus (KoRV) subtype expression over time reflects koala health outcomes. J Virol 93:e00849-e919

18. Chappell KJ, Brealey JC, Amarilla AA et al (2017) Phylogenetic diversity of koala retrovirus within a wild koala population. $\mathrm{J}$ Virol 91:e01820-e1916

19. Shimode S, Nakagawa S, Yoshikawa R, Shojima T, Miyazawa T (2014) Heterogeneity of koala retrovirus isolates. FEBS Lett 588:41-46

20. Oliveira NM, Farrell KB, Eiden MV (2006) In vitro characterization of a koala retrovirus. J Virol 80:3104-3107

21. Löber U, Hobbs M, Dayaram A et al (2018) Degradation and remobilization of endogenous retroviruses by recombination during the earliest stages of a germ-line invasion. Proc Natl Acad Sci USA 115:8609-8614

22. Johnson RN, O'Meally D, Chen Z et al (2018) Adaptation and conservation insights from the koala genome. Nat Genet 50:1102-1111

23. Kayesh MEH, Yamato O, Rahman MM et al (2019) Molecular dynamics of koala retrovirus infection in captive koalas in Japan. Arch Virol 164:757-765

24. Hashem MA, Kayesh MEH, Yamato O et al (2019) Coinfection with koala retrovirus subtypes $A$ and $B$ and its impact on captive koalas in Japanese zoos. Arch Virol 164:2735-2745

25. Sarker N, Fabijan J, Owen H et al (2020) Koala retrovirus viral load and disease burden in distinct northern and southern koala populations. Sci Rep 10:263

26. Bock M, Stoye JP (2000) Endogenous retroviruses and the human germline. Curr Opin Genet Dev 10:651-655
27. Boeke JD, Stoye JP (1997) Retrotransposons, endogenous retroviruses, and the evolution of retroelements. In: Coffin JM, Hughes $\mathrm{SH}$, Varmus HE (eds) Retroviruses. Cold Spring Harbor, Cold Spring Harbor Laboratory, New York, pp 343-346

28. Tsang J, Ribet D, Heidmann T, Dewannieux M (2019) Identification of the receptor used by the ecotropic mouse GLN endogenous retrovirus. J Virol 93:e01125-e1218

29. Shojima T, Hoshino S, Abe M, Yasuda J, Shogen H, Kobayashi T, Miyazawa T (2013) Construction and characterization of an infectious molecular clone of koala retrovirus. J Virol 87:5081-5088

30. Waugh C, Gillett A, Polkinghorne A, Timms P (2016) Serum antibody response to koala retrovirus antigens varies in free-ranging koalas (Phascolarctos cinereus) in Australia: implications for vaccine design. J Wildl Dis 52:422-425

31. Miyazawa T, Shojima T, Yoshikawa R, Ohata T (2011) Isolation of koala retroviruses from koalas in Japan. J Vet Med Sci 73:65-70

32. Fiebig U, Keller M, Denner J (2016) Detection of koala retrovirus subgroup B (KoRV-B) in animals housed at European zoos. Arch Virol 161:3549-3553

33. Fabijan J, Miller D, Olagoke O et al (2019) Prevalence and clinical significance of koala retrovirus in two South Australian koala (Phascolarctos cinereus) populations. J Med Microbiol 68:1072-1080

34. Legione AR, Patterson JLS, Whiteley P et al (2017) Koala retrovirus genotyping analyses reveal a low prevalence of KoRV-A in Victorian koalas and an association with clinical disease. J Med Microbiol 66:236-244

35. Sarker N, Fabijan J, Seddon J et al (2019) Genetic diversity of Koala retrovirus env gene subtypes: insights into northern and southern koala populations. J Gen Virol 100:1328-1339

36. Gao F, Yue L, Robertson DL et al (1994) Genetic diversity of human immunodeficiency virus type 2: evidence for distinct sequence subtypes with differences in virus biology. J Virol 68:7433-7447

37. Gao F, Bailes E, Robertson DL et al (1999) Origin of HIV-1 in the chimpanzee Pan troglodytes troglodytes. Nature 397:436-441

38. Simmons G, Clarke D, McKee J, Young P, Meers J (2014) Discovery of a novel retrovirus sequence in an Australian native rodent (Melomys burtoni): a putative link between gibbon ape leukemia virus and koala retrovirus. PLoS ONE 9:e106954

39. Alfano N, Michaux J, Morand S et al (2016) Endogenous gibbon ape leukemia virus identified in a rodent (Melomys burtoni subsp.) from Wallacea (Indonesia). J Virol 90:8169-8180

40. Hayward JA, Tachedjian M, Kohl C et al (2020) Infectious KoRVrelated retroviruses circulating in Australian bats. Proc Natl Acad Sci USA 117:9529-9536

41. Xu W, Eiden MV (2015) Koala retroviruses: evolution and disease dynamics. Annu Rev Virol 2:119-134

42. Tsangaras K, Siracusa MC, Nikolaidis N et al (2014) Hybridization capture reveals evolution and conservation across the entire koala retrovirus genome. PLoS ONE 9:e95633

43. Cui P, Löber U, Alquezar-Planas DE et al (2016) Comprehensive profiling of retroviral integration sites using target enrichment methods from historical koala samples without an assembled reference genome. PeerJ 4:e1847

44. Bendinelli M, Matteucci D, Friedman H (1985) Retrovirusinduced acquired immunodeficiencies. Adv Cancer Res 45:125-181

45. Bishop JM (1987) The molecular genetics of cancer. Science 235:305-311

46. Rosenberg N, Jolicoeur P (1997) Retroviral pathogenesis. In : Coffin JM et al (eds) Retroviruses. Cold Spring Harbour Laboratory Press

47. Tarlinton R, Meers J, Young P (2008) Biology and evolution of the endogenous koala retrovirus. Cell Mol Life Sci 65:3413-3421 
48. Fabijan J, Sarker N, Speight N et al (2020) Pathological findings in koala retrovirus-positive Koalas (Phascolarctos cinereus) from northern and southern Australia. J Comp Pathol 176:50-66

49. Butcher RG, Pettett LM, Fabijan J et al (2020) Periodontal disease in free-ranging koalas (Phascolarctos cinereus) from the Mount Lofty Ranges, South Australia, and its association with koala retrovirus infection. Aust Vet J 98:200-206

50. Fabijan J, Woolford L, Lathe S, Simmons G, Hemmatzadeh F, Trott DJ, Speight KN (2017) Lymphoma, koala retrovirus infection and reproductive chlamydiosis in a koala (Phascolarctos cinereus). J Comp Pathol 157:188-192

51. Carey AJ, Timms P, Rawlinson G, Brumm J, Nilsson K, Harris JM, Beagley KW (2010) A multi-subunit chlamydial vaccine induces antibody and cell-mediated immunity in immunized koalas (Phascolarctos cinereus): comparison of three different adjuvants. Am J Reprod Immunol 63:161-172

52. Kollipara A, George C, Hanger J, Loader J, Polkinghorne A, Beagley K, Timms P (2012) Vaccination of healthy and diseased koalas (Phascolarctos cinereus) with a Chlamydia pecorum multisubunit vaccine: evaluation of immunity and pathology. Vaccine 30:1875-1885

53. Denner J, Norley S, Kurth R (1994) The immunosuppressive peptide of HIV-1: functional domains and immune response in AIDS patients. Aids 8:1063-1072

54. Ishida Y, McCallister C, Nikolaidis N, Tsangaras K, Helgen KM, Greenwood AD, Roca AL (2015) Sequence variation of koala retrovirus transmembrane protein $\mathrm{p} 15 \mathrm{E}$ among koalas from different geographic regions. Virology 475:28-36

55. Fiebig U, Keller M, Möller A, Timms P, Denner J (2015) Lack of antiviral antibody response in koalas infected with koala retroviruses (KoRV). Virus Res 198:30-34

56. Olagoke O, Quigley BL, Eiden MV, Timms P (2019) Antibody response against koala retrovirus (KoRV) in koalas harboring KoRV-A in the presence or absence of KoRV-B. Sci Rep 9:12416

57. Abts KC, Ivy JA, DeWoody JA (2015) Immunomics of the koala (Phascolarctos cinereus). Immunogenetics 67:305-321

58. Maher IE, Griffith JE, Lau Q, Reeves T, Higgins DP (2014) Expression profiles of the immune genes CD4, CD8 $\beta$, IFN $\gamma$, IL-4, IL-6 and IL-10 in mitogen-stimulated koala lymphocytes (Phascolarctos cinereus) by qRT-PCR. PeerJ 2:e280

59. Lu W, Mehraj V, Vyboh K, Cao W, Li T, Routy JP (2015) $\mathrm{CD} 4: \mathrm{CD} 8$ ratio as a frontier marker for clinical outcome, immune dysfunction and viral reservoir size in virologically suppressed HIV-positive patients. J Int AIDS Soc 18:20052

60. Serrano-Villar S, Sainz T, Lee SA et al (2014) HIV-infected individuals with low $\mathrm{CD} 4 / \mathrm{CD} 8$ ratio despite effective antiretroviral therapy exhibit altered $\mathrm{T}$ cell subsets, heightened CD8+ T cell activation, and increased risk of non-AIDS morbidity and mortality. PLoS Pathog 10:e1004078

61. Olagoke O, Miller D, Hemmatzadeh F et al (2018) Induction of neutralizing antibody response against koala retrovirus (KoRV) and reduction in viral load in koalas following vaccination with recombinant KoRV envelope protein. NPJ Vaccines 3:30

62. Puls RL, Emery S (2006) Therapeutic vaccination against HIV: current progress and future possibilities. Clin Sci (Lond) 110:59-71

63. Groschel MI, Prabowo SA, Cardona PJ, Stanford JL, van der Werf TS (2014) Therapeutic vaccines for tuberculosis-a systematic review. Vaccine 32:3162-3168

64. Waugh C, Khan SA, Carver S et al (2016) A prototype recombinant-protein based chlamydia pecorum vaccine results in reduced chlamydial burden and less clinical disease in free-ranging koalas (Phascolarctos cinereus). PLoS ONE 11:e0146934

65. Kita Y, Hashimoto S, Nakajima T et al (2013) Novel therapeutic vaccines [(HSP65+IL-12)DNA-, granulysin- and Ksp37-vaccine] against tuberculosis and synergistic effects in the combination with chemotherapy. Hum Vaccin Immunother 9:526-533

66. Jindra C, Huber B, Shafti-Keramat S et al (2015) Attenuated recombinant influenza A virus expressing HPV16 E6 and E7 as a novel therapeutic vaccine approach. PLoS ONE 10:e0138722

67. Messer RJ, Dittmer U, Peterson KE, Hasenkrug KJ (2004) Essential role for virus-neutralizing antibodies in sterilizing immunity against Friend retrovirus infection. Proc Natl Acad Sci USA 101:12260-12265

68. Vaine M, Lu S, Wang S (2009) Progress on the induction of neutralizing antibodies against HIV type 1 (HIV-1). BioDrugs 23:137-153

69. Denner J (2013) Immunising with the transmembrane envelope proteins of different retroviruses including HIV-1: a comparative study. Hum Vaccin Immunother 9:462-470

70. Osterhaus A, Weijer K, UytdeHaag F, Knell P, Jarrett O, Akerblom L, Morein B (1989) Serological responses in cats vaccinated with FeLV ISCOM and an inactivated FeLV vaccine. Vaccine 7:137-141

71. Fiebig U, Dieckhoff B, Wurzbacher C, Möller A, Kurth R, Denner J (2015) Induction of neutralizing antibodies specific for the envelope proteins of the koala retrovirus by immunization with recombinant proteins or with DNA. Virol J 12:1-8

72. Tsukiyama-Kohara K, Kohara M (2014) Tupaia belangeri as an experimental animal model for viral infection. Exp Anim 63:367-374

73. Xu W, Gorman K, Santiago JC, Kluska K, Eiden MV (2015) Genetic diversity of koala retroviral envelopes. Viruses $7: 1258-1270$

Publisher's Note Springer Nature remains neutral with regard to jurisdictional claims in published maps and institutional affiliations. 